\title{
Measurement of the internal size of bronchi using high resolution computed tomography (HRCT)
}

\author{
E. Senéterre*, F. Paganin**, J.M. Bruel*, F.B. Michel**, J. Bousquet**
}

Measurement of the internal size of bronchi using high resolution computed tomography (HRCT). E,. Senéterre, F. Paganin, J.M. Bruel, F.B. Michel, J. Bousquet. CERS Journals Ltd 1994.

ABSTRACT: Computed tomography (CT) of the lungs makes it possible to study indindual airways. A technique using high-resolution-CT (HRCT) has been developed to measure the internal size of the bronchi.

Five normal subjects had a conventional HRCT scan at full, deep inspiration. The internal size of the airways from segmental to the sixth generation bronchi was measured using an original method of data analysis.

The size of the airways ranged $0.5-50 \mathrm{~mm}^{2}$ (approximatively $0.8-8 \mathrm{~mm}$ in diameter.

The technique was found to be highly reproducible, making it possible to study the variation in airways calibre after provocative challenge or administration of bronchodilator in asthma.

Eur Respir J., 1994, 7, 596-600.
*Service de Radioiogie, Hôpital St Eloi, Montpellier, France. **Services des Maladies Respiratoires, Hôpital Amaud de Villeneuve, Montpellier, France.

Correspondence: J. Bousquet Clinique des Maladies Respiratoires Hôpital Amnud de Viileneuve Centre Hôspitalier Universitaire 34059 Montpeilier Cedex France

Keywords: Bronchus computed tomography size

Received: December 261992

Accepted after revision October 31993
In asthma, computed tomographic (CT)-scan has made it possible to visualize reversible and irreversible airways abnormalities that are not seen using conventional roentgenograms $[1,2]$. Due to its high cost, CT-scan cannot be used as a routine procedure for the assessment of the severity of disease in asthmatic patients, but it can be considered as a useful tool for examining airways lesions in patients in whom asthma is not fully controlled.

CT-scans allow individual airways to be studied, and can thus be used to measure changes in bronchial calibre following antigen challenge or bronchodilator treatment. This technique is very sensitive and it has been demonstrated that bronchi as small as $2 \mathrm{~mm}$ in diameter can be accurately measured [3]. In 1984, WeBв et al. [4] set out to determine the most appropriate method for the visualization and measurement of bronchi, and found that a window level of -450 Hounsfield units (HU) was most appropriate. High resolution (HR)-CT has only recently been introduced as an in vivo technique and was used by Brown et al. [5] and Herold et al. [6] to measure the internal diameter of bronchi in dogs.

In this study, we describe an original technique using CT-scan to measure the internal size of the bronchi in normal human subjects, and we have assessed the reproducibility of the method.

\section{Materials and methods}

\section{Subjects}

Five healthy male subjects (17-32 yrs, mean \pm sD $26 \pm 5.5 \mathrm{yrs}$ ) were studied. None or them was a current smoker or had a history of chest disease or bronchial hyperactivity, and their pulmonary function was normal forced expiratory volume in one second $\left(\mathrm{FEV}_{1}\right) 100 \pm 4 \%$ of predicted values.

\section{CT-scan}

CT scans of the chest were performed with a Somatom DRH scanner (Siemens, Erlangen, Germany) in high resolution technique, according to MAYO et al. [7] using the following parameters: $125 \mathrm{kV}, 310 \mathrm{mAs}$, matrix size of $512 \times 512$, slice thickness of $1 \mathrm{~mm}$, interslice gap of 3 $\mathrm{mm}$, scan time of $4 \mathrm{~s}$ and inter-scan time of $8 \mathrm{~s}$.

Subjects were asked to take a maximal inspiration and maintain the breathhold as long as possible. A series of slices were performed during each breathhold. The number of slices by series ranged from 4-14 (average 8). Scan levels were chosen to give images of bronchi having a cranio-caudal orientation in the upper and lower lobes. For the upper lobes, scans were started $15 \mathrm{~mm}$ above the carina and millimetric slices were taken upward. For the lower lobes, scans were started at a point $60 \mathrm{~mm}$ below the carina and millimetric slices were taken downward. The numbers of slices series ranged from $2-4$ for each lobe. The starting point for the further series was set at the same level of the antepenultimate slice of the previous series making it possible to compare both series.

The skin radiation dose is $41.5 \mathrm{mGy}$ per slice, and no more than two slices were laken at the same level.

\section{Data analysis}

Selection af slices to be studied. Comparable slice levels were identified using anatomical landmarks, such as 
airways or vascular branching points, as described by BROwn et al. [5] and McNamara et al. [8]. For each pair of slices at a comparable level, the transverse and anteroposterior diameters of the chest were compared, to assess whether the two separate inspirations resulted in alterations in chest volume. The maximal acceptable variation for either measurement was taken as $3 \%$.

Reconstruction of the image of the selected slices. In order to analyse the size of the bronchi, a reconstruction of the image was obtained from the data-base with a zoom factor of 10 and a field view of $5.1 \mathrm{~cm}$, focused on the bronchi to be analysed. The pixel size was around $100 \mu \mathrm{m}$. The estimated spatial resolution was $550 \mu \mathrm{m}$

a)

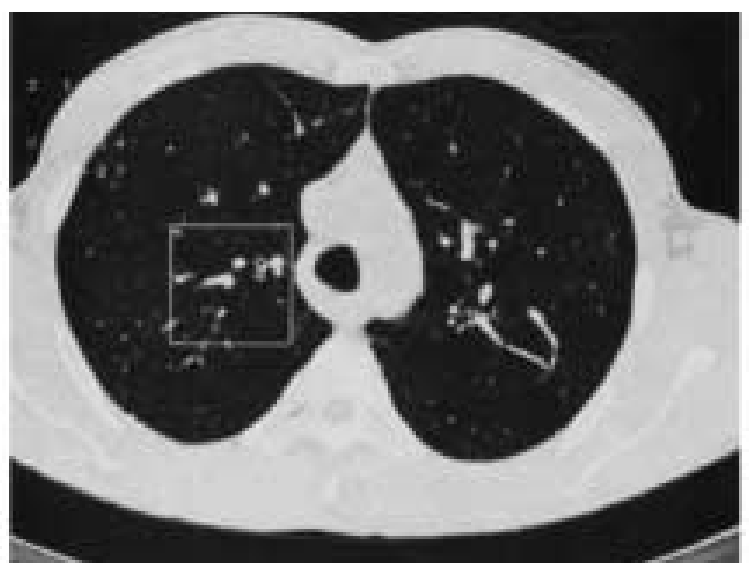

c)
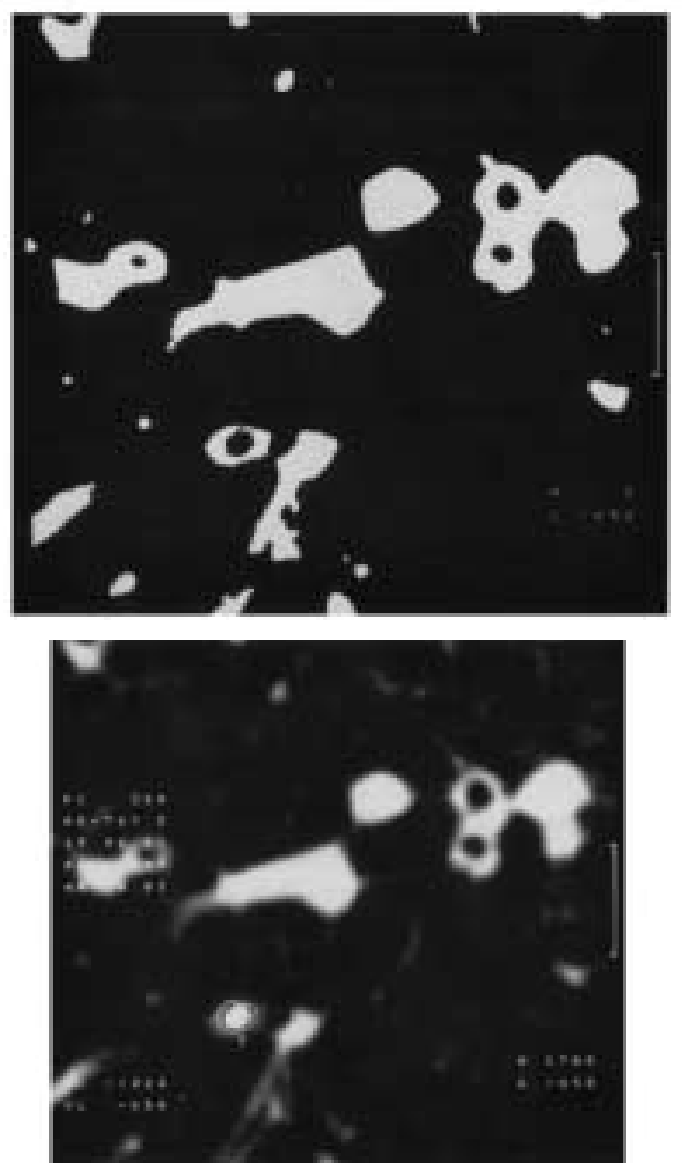

(data kindly provided by Siemens) and is in accordance with the data of MurATA et al. [3].

Selection of the bronchi to be studied. Bronchi were excluded from analysis if they were oriented in an oblique fashion, if branching occurred at the level chosen, or if artefacts were seen to be present. An index of airway roundness was determined using the method of McNAMARA et al. [8]. The largest luminal diameter (DL) was measured as was the largest muminal diameter perpendicular to DL (DS). When the ratio of DL/DS was equal to or greater than 1.5 , the bronchus was considered to be obliquely orientated and was excluded. Artefacts included blurred or irregular contours of the bronchi as well as lack of homogeneity of the lung parenchyma.

b)

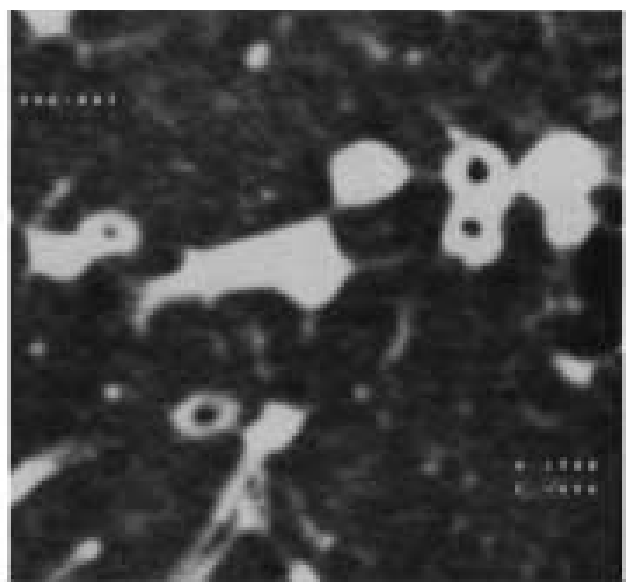

d)

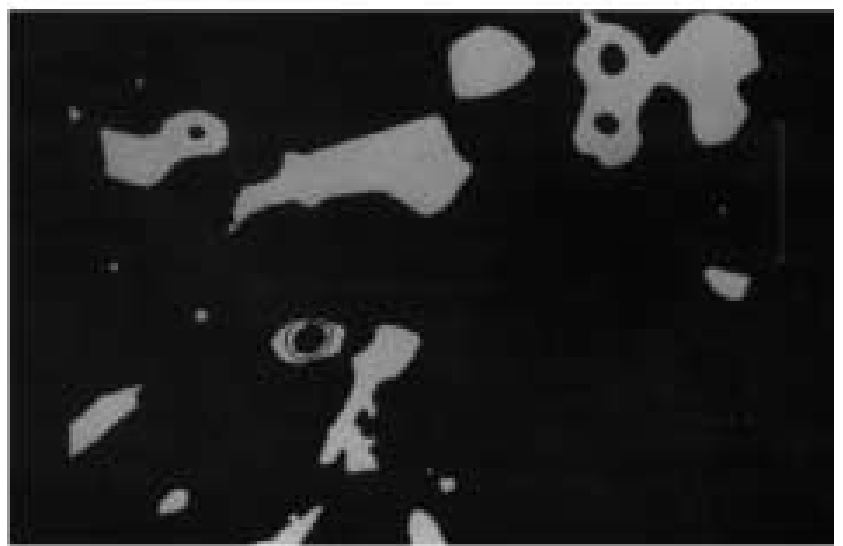

Fig. 1. - Method for the measurement of the internal size of a bronchus; a) selection of the slice using anatomical landmarks, (the square indicates the area to be enlarged); b) reconstruction on 5.1 field of view; c) determination of the higher window level, which obtains a circular representation of each bronchus with a window width of $2 \mathrm{HU}$; d) drawing of the region of interest (ROI) on the bronchial wall; e) measure of the luminal area (pixels: $\mathrm{PC}=380$ ). 
Comparative measurement of the internal size of the bronchi. For the two images of the same bronchus the window width was set to the minimum value (2 HU), in order to get a black and white representation of the bronchus. A region of interest (ROI) was drawn on the bronchus wall, and the luminal area was measured by computed calculation as the number of pixels in the ROI having attenuation values under the window level. This level, called discrimination level, was determined as the highest window level which obtains a closed circle as representation of the bronchus for the two images (fig. 1). Therefore, a separate discrimination level was identified for each bronchus analysed. The internal diameter of the airways can be derived approximately calculated from the formula: Area $=\pi r^{2}$.

\section{Reproducibility of the measurements}

Reproducibility of data analysis was studied by the measurement of the internal area of the bronchi carried

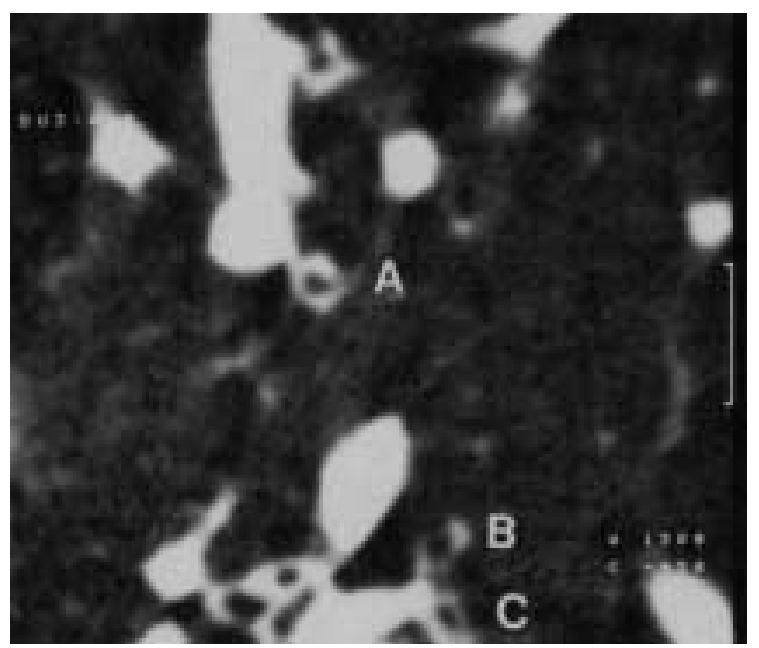

Fig. 2. - Examples of artefacts inhomogeneous density of bronchial wall (bronchus A and B); irregular contours (bronchus A); excessive blurring of edges (bronchus B); obliquely orientated bronchus (bronchus c); heterogeneity of the lung parenchyma (left part of the picture). out twice by the same observer (intraobserver reproducibility), and by two different observers (interobserver reproduciblity). The reproducibility of the discrimination level determination was carried out by two different observers. The reproducibility of data acquisition was assessed by the comparison of the luminal area of the same bronchus, studied in two slices performed at the same level during two experiment series. Statistical analysis was performed using Spearman's rank test.

To examine the importance of respiration phases, we studied two patients at full, deep inspiration and at tidal volume.

\section{Results}

Using HRCT-scan it is possible to measure the internal size of the airways from segmental to sixth generation bronchi. Three to 11 bronchi were analysed for each lobe (mean \pm SD 5.6 \pm 2.3 ). The discrimination level used in this study was not constant and ranged from -349 to $-950 \mathrm{HU}$ (mean \pm SD $-737 \pm 127 \mathrm{HU}$ ). The luminal area ranged from 54 to 5,028 pixels (mean \pm SD: $766 \pm 868$ pixels), corresponding to a mean area of $7.7 \pm 8.7 \mathrm{~mm}^{2}$ (i.e. $0.8-8 \mathrm{~mm}$ in luminal diameter).

Measurement of the luminal area was performed in two subjects at full deep inspiration and at tidal volume. At tidal volume, blurring of bronchial contours hypodense and hyperdense bands of heterogeneity resulting in inhomogeneity of density of the bronchial wall and the parenchyma were seen much more frequently than at full, deep inspiration. These artefacts resemble those secondary to the motion of the aortic cross in the left upper lobe. Nonhomogeneous density of the bronchial wall and blurring of contours made it impossible to accurately measure the lumen of the bronchi.

Artefacts included blurred or irregular contours of the bronchi, as well as heterogeneity of lung parenchyma (fig. 2).

For all measurements studied the reproducibility was excellent and highly significant (figs. 3 and 4). a)

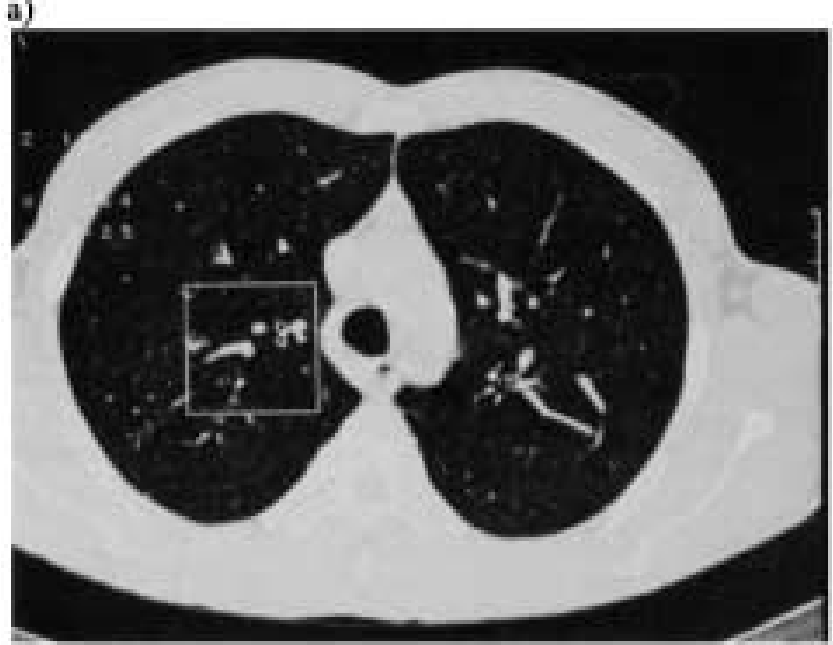

b)

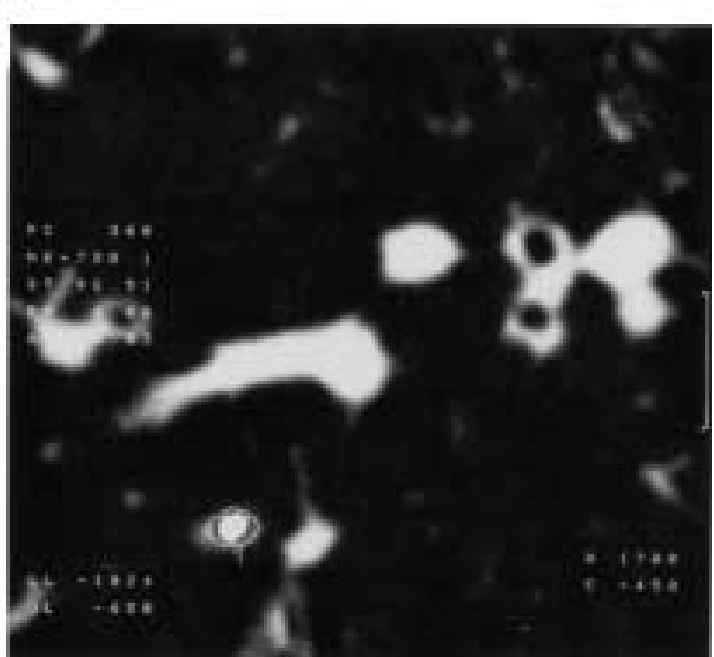

Fig. 3. - Reproducibility of the technique. The area of the chest shown on figure 1 was reconstructed by a second investigator. a) selection of the slice using anatomic landmarks, (the square indicates the area to be enlarged); b) measure of the luminal area (pixel count: PC $=360$ ). 

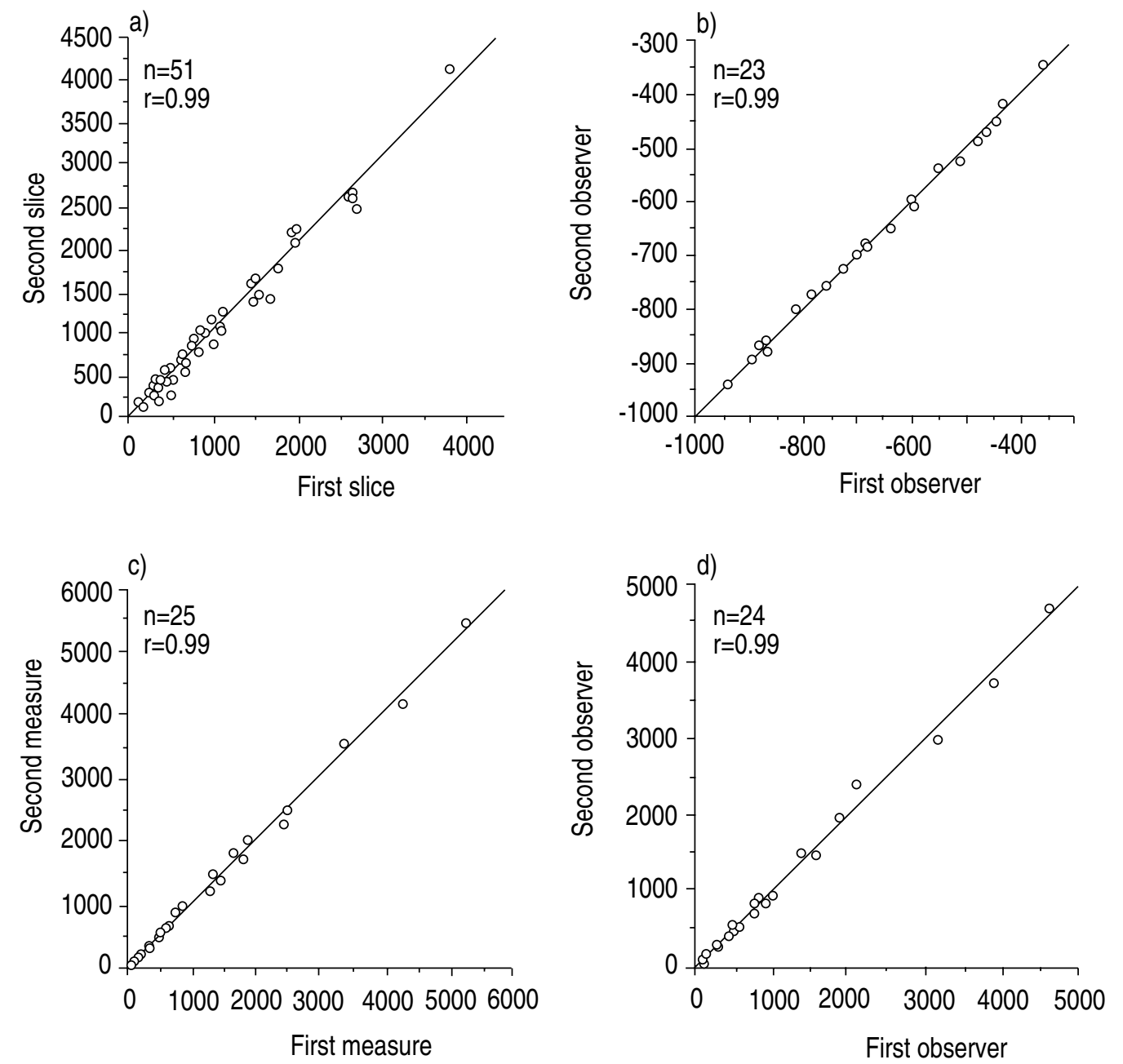

Fig. 4. - Reproducibility of the technique; statistical analyses by Spearman's rank test. a) reproducibility of the measure of the same bronchus studied in two slices performed at the same level (pixel numbers); b) reproducibility of discrimination level assessed by two observers (Hounsfield units); c) intraobserver reproducibility (pixel numbers); d) interobserver reproducibility (pixel numbers).

\section{Discussion}

The technique used in this paper is original and was not directly derived from any previous study performed in either animals or in man. Although the methods described are relatively simple, care must be taken to carry them out precisely.

One major pitfall in the measurement of the internal diameter of the bronchi is that it may be affected by the phases of inspiration. HRCT-scans should be performed whilst the patient is in full, deep inspiration, in accordance with the recent guidelines on the use of HRCTscans in the lung [9]. However, measurement of bronchial size should be carried out during this phase of inspiration not only because it conforms to these guidelines but also because at tidal volume an increased number of artefacts are observed, including excessive blurring at the edges and lack of homogeneity of the bronchial wall. Moreover, a full deep inspiration is easier to reproduce. It is possible to measure the maximum inspiration using a method described by KALENDER et al. [10]. However, the reproducibility of full deep inspiration is sufficiently high $(2-3 \%)$ [11] that individual measurements are not warranted. The anteroposterior and lateral diameters of the ribcage were measured for each pair of slices, and the measurements never varied by more than $3 \%$. Since the variation of pulmonary volume can be considered to be isotropic, the variation in thoracic volume cannot exceed $10 \%$. Thus, it is unlikely that such small variations could significantly influence the size of the bronchi.

Since this method is primarily aimed at studying the effects of bronchial challenge or bronchodilator treatments on bronchial size, three factors should be borne in mind in order to achieve the best possible comparison between slices performed at different time points. Firstly, it is necessary to perform the slices at exactly the same level. This can be achieved by locating anatomical landmarks as previously described by other researchers [5, 8] and confirmed by us in this study. Secondly, the method of measurement of the airways 
size should avoid operator subjectivity. This drawing of the ROI on the bronchial wall can be easily performed when the window width is minimal (2 HU) since there is high contrast and the wall and the lumen can be clearly distinguished. In addition, provided that the ROI drawing does not cross the boundary of the lumen, there is no operator influence on the calculation of the luminal area. We examined the interobserver variability and found highly reproducible measurements of the bronchial size, indicating that the technique is independent of the skill of the investigator. Thirdly, the same window level has to be used for the serial measurement of the same bronchus. However, although some authors have used a single window level for all the bronchial measurements, we used variable levels for the different bronchi. McNAMARA et al. [8] and WЕвв et al. [4] determined the optimal window level to be $-450 \mathrm{HU}$, using phantoms with a wall thickness, respectively, of $0.5-2.3 \mathrm{~mm}$ and $1.5-1.8$ $\mathrm{mm}$. However, the wall thickness of bronchi varies from major bronchi down to the smallest ones; of the latter group the smallest discernable size determined by HRCTscan is $0.1 \mathrm{~mm} \mathrm{[3].} \mathrm{Using} \mathrm{a} \mathrm{single} \mathrm{discrimination} \mathrm{level}$ of $-450 \mathrm{HU}$, it is impossible to measure small bronchi because the wall density is below this window level. In the present study, bronchi had wall densities ranging from $-950 \mathrm{HU}$ for the smallest bronchi to $-349 \mathrm{HU}$ for the largest ones. Moroever, we did not use a single level of $-950 \mathrm{HU}$ for all bronchi, since at this discrimination level some bronchi appeared to be closed, emphasizing the usefulness of a variable window level.

HRCT can, therefore, be used to measure bronchial luminal area with a simple method when patients are at full, deep inspiration. Further studies must, however, be performed to correlate the results of the measurements with the real size of bronchi, using morphometric studies [8]. This technique was found to be highly reproducible and may be useful in studying the variation of the airways calibre either during challenge or after bronchodilator therapy, and in assessing the heterogeneity of the airways obstruction.

\section{References}

1. Paganin F, Trussard V, Senéterre E, et al. Chest radiography and high-resolution computed tomography of the lungs in asthma. Am Rev Respir Dis 1992; 146: 1084-1087.

2. Kinsella M, Muller NL, Staoles C, Vedal S, Chan-Yeung M. Hyperinflation in asthma and emphysema: assessment by pulmonary function testing and computed tomography. Chest 1988; 94: 286-289.

3. Murata K, Itoh $\mathrm{H}$, Todo $\mathrm{G}$, et al. Centrilobular lesions of the lung: demonstralion by high-resolution CT and pathologic correlation. Radiology 1986; 161: 641-645.

4. Webb RW, Gamsu G, Wall SD, et al. CT of a bronchial phantom: factors affecting appearence and size measurements. Invest Radiol 1984; 19: 394-398.

5. Brown RH, Herold CJ, Hirshman CA, et al. In vivo measurements of airway reactivity using high-resolution computed tomography. Am Rev Respir Dis 1991; 144: 208-212.

6. Herold CJ, Brown RH, Mitzner W, Links JM, Hirshman CA, Zerhouni EA. Assessment of pulmonary hypereactivity with high-resolution CT. Radiology 1991; 181: 369-374.

7. Mayo JR, Webb WR, Gould R et al. High-resolution CT of the lungs: an optimal approach. Radiology 1987; 163: 507-510.

8. McNamara AE, Muller NL, Okazawa M, Arntorp J, Wiggs $\mathrm{BR}$, Pare PD. Airway narrowing in excised canine lungs measured by high-resolution computed tomography. J Appl Physiol 1992; 73: 307-316.

9. Swensen SJ, Aughenbaugh GL, Douglas WW, Myers JL. High-resolution CT of the lungs: Findings in various pulmonary diseases. Am J Roentgenol 1992; 158: 971-997.

10. Kalender WA, Reimüller R, Seissler W, Behr J, Welke M, Fichke H. Measurement of pulmonary parenchymal attenuation: use of spirometric gating with quantitative CT. Radiology 1990; 175: 265-268.

11. Quanjer PH. Standardized lung function testing. Bull Eur Physiopathol Respir 1983; 195: 1-95. 